\title{
Calores específicos dos gases ideais degenerados
}

\author{
Specific heats of degenerate ideal gases \\ Francisco Caruso*1, Vitor Oguri², Felipe Silveira ${ }^{2}$ \\ ${ }^{1}$ Centro Brasileiro de Pesquisas Fisicas, Rio de Janeiro, RJ, Brasil. \\ ${ }^{2}$ Universidade do Estado do Rio de Janeiro, Departamento de Física Nuclear e Altas Energias, Rio de Janeiro, RJ, Brasil.
}

Recebido em 26 de Abril, 2017. Revisado em 29 de Junho, 2017. Aceito em 07 de Agosto, 2017.

\begin{abstract}
A partir de argumentos baseados no princípio da incerteza de Heisenberg e no princípio de exclusão de Pauli, estimam-se os calores específicos molares dos gases ideais degenerados em baixas temperaturas, com resultados compatíveis com o princípio de Nerst-Planck (a 3 a lei da Termodinâmica). É apresentado, ainda, o fenômeno da condensação de Bose-Einstein com base no comportamento do calor específico de gases de bósons massivos e não relativísticos.
\end{abstract}

Palavras-chave: calor específico, gases degenerados, condensação de Bose-Einstein.

From arguments based on Heisenberg's uncertainty principle and Pauli's exclusion principle, the molar specific heats of degenerate ideal gases at low temperatures are estimated, giving rise to values consistent with the Nerst-Planck Principle (third law of Thermodynamics). The Bose-Einstein condensation phenomenon based on the behavior of specific heat of massive and non-relativistic boson gases is also presented.

Keywords: specific heat, degenerate gases, Bose-Einstein condensation.

\section{Introdução}

Após um longo tempo de ensino dos fenômenos térmicos em cursos de Física, é fácil perceber a necessidade de uma abordagem que facilite aos alunos a transição da Termodinâmica para a Física Estatística. As dificuldades podem ser percebidas em disciplinas tais como Física Moderna, Estrutura da Matéria e Mecânica Estatística.

A abordagem estatístico-probabilística, tanto em seus aspectos formais como conceituais, não é do domínio da maioria dos alunos, mesmo daqueles que já superaram o ciclo básico do ensino superior. Por exemplo, muitos estudantes têm dificuldades em compreender a explicação do calor específico dos gases e dos sólidos baseada no princípio da equipartição de energia, pois ainda não sabem utilizar a distribuição de Maxwell-Boltzmann, ou qualquer distribuição de probabilidades, para o cálculo de valores médios ${ }^{1}$ Desta forma, procura-se apresentar um método de se chegar aos resultados conhecidos sem recorrer a conceitos probabilísticos.

O calor específico expressa a capacidade de uma substância absorver energia quando excitada por algum agente externo. Quanto maior o número de modos pelos quais é possível essa absorção maior o calor específico de uma substância. Por esse motivo, o calor específico de um gás monoatômico é menor do que a de um gás poliatômico, ou de um sólido.

\footnotetext{
*Endereço de correspondência: caruso@cbpf.br

${ }^{1}$ Essa mesma dificuldade, por mais estranha que pareça, é encontrada na Mecânica Quântica, após a interpretação probabilística de Born.
}

Desde sua descoberta 1], os estudos e as medidas dos calores específicos têm contribuído de forma determinante para a compreensão da estrutura da matéria. Por exemplo, as medidas dos calores específicos dos sólidos por P.L. Dulong e A.T. Petit (1819) 2 permitiram que J.J. Berzelius 3] corrigisse o peso atômico de vários elementos químicos ao longo do século XIX. Esse trabalho sistemático do químico sueco foi fundamental para que D. Mendeleiev [4] pudesse elaborar a sua Tabela Periódica, em torno de 1869.

Embora para gases monoatômicos os primeiros resultados, baseados no princípio da equipartição da energia, tenham sido satisfatórios, o mesmo não ocorreu para líquidos, sólidos e gases de moléculas mais complexas. A comprovação de que os calores específicos variavam com a temperatura, ao contrário da lei de Dulong-Petit, exigiu a revisão crítica de vários conceitos físicos, levando a modificações profundas, não dos fundamentos da Física Estatística, como se acreditava, mas da própria Mecânica Clássica [5]. Foram as medidas desses calores específicos dos sólidos a baixas temperaturas que permitiram testar a então nova teoria atômica da matéria.

As bases da Física Estatística foram estabelecidas por L. Boltzmann (1884) [6] e J. W. Gibbs (1901) [7], a partir do conceito clássico de estado de um sistema como pontos de um contínuo. No entanto, a descoberta de Planck (1900) [8], generalizada pela Mecânica Quântica [9], de que os estados de um sistema, confinado em um volume, constituem um conjunto discreto associado a um espectro discreto de energia, implica a discretização do próprio 
espaço de fase, independentemente de qualquer conceito estatístico.

Do ponto de vista quântico, as partículas de um sistema, em baixas temperaturas, tendem a se agrupar pelos estados associados aos menores valores de energia. Nesse limite há uma maior organização e uma diminuição da capacidade de excitação do sistema, o que implica decréscimo da entropia e do calor específico de um sistema. Esse é o conteúdo da 3 â lei da Termodinâmica 10 15 ${ }^{2}$

Como é mostrado nesse artigo, a definição de quantidades e parâmetros - como densidade de estados, temperatura de degenerescência - característicos de um gás ideal, baseada em princípios quânticos, permite estabelecer a divisão dos gases em duas categorias: os gases de férmions e os gases de bósons. A partir dessa divisão, obtêm-se estimativas para os calores específicos dos gases ideais em baixas temperaturas, compatíveis com a 3 a lei da Termodinâmica.

Ao final do artigo, com base no comportamento do calor específico de um gás de bósons massivos e não relativísticos, o fenômeno da condensação de Bose-Einstein é apresentado como uma transição de fase para um estado mais ordenado, o condensado de Bose-Einstein.

\section{Limites dos gases ideais}

Os gases moleculares em condições ambientais têm densidades que variam da ordem de $10^{-5} \mathrm{~g} / \mathrm{cm}^{3}$ a $10^{-3} \mathrm{~g} / \mathrm{cm}^{3}$, e se comportam como gases ideais para os quais a energia interna depende apenas da temperatura e o calor específico molar é constante 12 15].

Sendo $N$ o número total das partículas (átomos ou moléculas) constituintes de um gás ideal, e $G$ o número de estados associados às partículas ${ }^{3}$ a razão $(N / G)$ entre esses números permite a divisão dos gases ideais em duas classes 12,16]: não degenerados e degenerados.

$$
\begin{cases}N / G \ll 1 & \text { (gases não degenerados) } \\ N / G \geq 1 & \text { (gases degenerados) }\end{cases}
$$

Enquanto as propriedades dos gases não degenerados não dependem da natureza de suas partículas constituintes, os gases degenerados evidenciam a natureza quântica de suas partículas, a qual se reflete em seu comportamento macroscópico.

Em geral, o comportamento degenerado manifestase em baixas temperaturas ou altas densidades de um gás, quando as partículas têm de competir pelos estados acessíveis. Em altas temperaturas ou baixas densidades, quando o número de estados acessíveis é muito maior

\footnotetext{
${ }^{2}$ A $3^{3}$ a lei da Termodinâmica, ou lei de Nernst, estabelece que a entropia de um sistema se aproxima de um valor constante no limite $T \rightarrow 0$, o qual, segundo Planck, é nulo. Desse modo, o calor específico também é nulo quando $T=0$.

3 O estado de uma partícula é caracterizado por valores de grandezas como a posição e o momentum, ou energia.
}

que o número de partículas, a competição pelos estados praticamente não existe, e o gás exibe comportamento não degenerado.

\section{Número e densidade de estados}

Utilizando-se uma abordagem semiclássica para o cálculo do número de estados acessíveis às partículas quase livres de um gás ${ }^{4}$ contidas em um volume $V$ e com momenta que variam de zero a um dado valor $p$, os limites que caracterizam o comportamento dos gases ideais podem ser apropriadamente quantificados.

Do ponto de vista da Mecânica Clássica [7:13], o estado de uma única partícula é caracterizado por sua posição e momentum. Assim, a evolução ao longo do tempo do estado da partícula pode ser representada em um espaço de dimensão seis, denominado espaço de fase da partícula, no qual cada ponto de coordenadas $\left(x, y, z, p_{x}, p_{y}, p_{z}\right)$ caracteriza o estado dinâmico dessa partícula.

Se a partícula move-se ao longo de uma direção $x$, entre 0 e $L$, em qualquer sentido, com momentum menor que um valor $p$, sua evolução é visualizada em um plano no qual cada ponto $\left(x, p_{x}\right)$, dentro do retângulo de lados $L$ e $2 p$ (Figura 1), representa um possível estado acessível à partícula. A área desse retângulo é proporcional ao número de estados acessíveis à partícula.

Nesse contexto, segundo argumento originalmente proposto por O. Sackur e H. Tetrode [17] em 1912 5 o número de estados $(G)$ acessíveis a cada uma das partículas de um gás contido em um recipiente de volume $V$ pode ser expresso pela razão entre o volume no espaço de fase de dimensão seis, associado a uma única partícula, cujo momentum varia de zero até um valor $p$, e um volume

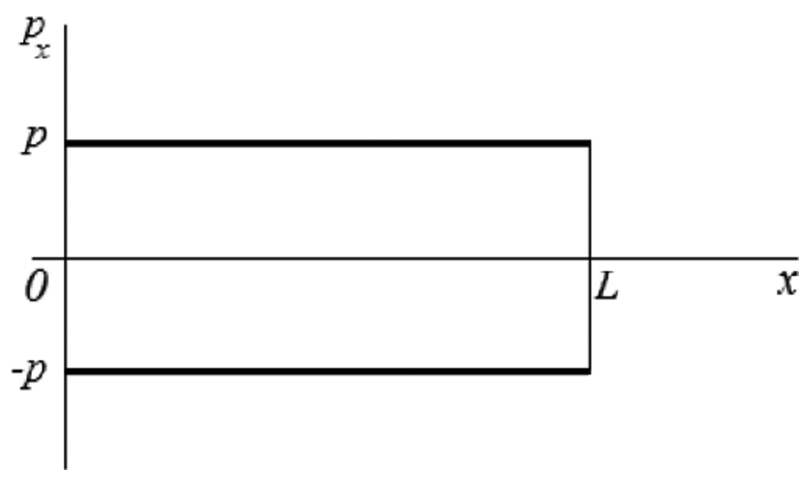

Figura 1: Plano de fase de uma partícula que se desloca ao longo da direção $x$ com momentum entre $-p$ e $p$, confinada no intervalo $(0, L)$.

\footnotetext{
${ }^{4} \mathrm{O}$ intervalo de tempo da interação de uma partícula de um gás com outras partículas é bem pequeno em relação ao intervalo de tempo no qual que a partícula se move livremente.

${ }^{5}$ Esse argumento foi utilizado também por S. Bose 18 em 1924, ao deduzir a fórmula de Planck para a radiação de corpo negro.
} 
mínimo igual a $h^{3}$,

$$
\begin{array}{r}
G=f \frac{1}{h^{3}} \iiint \iiint \mathrm{d} x \mathrm{~d} y \mathrm{~d} z \mathrm{~d} p_{x} \mathrm{~d} p_{y} \mathrm{~d} p_{z}= \\
f \frac{V}{h^{3}}\left(\frac{4 \pi}{3} p^{3}\right),
\end{array}
$$

sendo $h \simeq 6,626 \times 10^{-34} \mathrm{~J} \cdot \mathrm{s}$ a constante de Planck. O fator $f$ depende da natureza das partículas $\sqrt{6}$ e o termo entre parênteses é o volume da esfera de raio $p=\sqrt{p_{x}^{2}+p_{y}^{2}+p_{z}^{2}}$ no espaço dos momenta.

A existência de um volume mínimo no espaço de fase pode ser justificado a partir do princípio da incerteza de Heisenberg [5, 9, 12], o qual estabelece uma correlação entre o momentum $\left(p_{x}\right)$ e a posição $(x)$ de uma partícula em uma dada direção, tal que o limite mínimo para o produto das incertezas $\left(\Delta_{x}, \Delta_{p_{x}}\right)$ associadas às medidas desses pares de variáveis é da ordem da constante de Planck.

Para as três direções espaciais, pode-se escrever

$$
\begin{aligned}
& \left(\Delta_{x} \cdot \Delta_{p_{x}}\right)_{\min } \sim h, \\
& \left(\Delta_{y} \cdot \Delta_{p_{y}}\right)_{\min } \sim h, \\
& \left(\Delta_{z} \cdot \Delta_{p_{z}}\right)_{\min } \sim h .
\end{aligned}
$$

De acordo com as relações entre o momentum $(p)$ e a energia $(\epsilon)$ de uma partícula livre,

$$
\begin{cases}p=\sqrt{2 m \epsilon} \quad & \text { (partículas não relativísticas } \\ & \text { de massa } m \text { ) } \\ & \\ & \text { (partículas ultrarrelativísticas ou } \\ & \text { não massivas com velocidade } c: \\ & \text { fótons, fônons, ...) }\end{cases}
$$

pode-se expressar o número de estados acessíveis eq. (2) a cada partícula de um gás como função da energia por

$$
G(\epsilon)=\left\{\begin{array}{l}
\left(\frac{4 \pi f}{3}\right) \frac{V}{h^{3}}(2 m \epsilon)^{3 / 2} \\
\left(\frac{4 \pi f}{3}\right) \frac{V}{(c h)^{3}} \epsilon^{3}
\end{array}\right.
$$

Para um gás molecular nas condições ambientais, o número de estados acessíveis é da ordem de $10^{27}$ e o número de moléculas, cerca de $10^{23}$. Nessas condições, o estado do gás é não degenerado.

Definindo-se a densidade de estados $g(\epsilon)$ de uma partícula livre por

$$
g(\epsilon)=\frac{\mathrm{d} G}{\mathrm{~d} \epsilon}=\left\{\begin{array}{l}
2 \pi f V\left(\frac{2 m}{h^{2}}\right)^{3 / 2} \epsilon^{1 / 2}, \\
4 \pi f \frac{V}{(c h)^{3}} \epsilon^{2}
\end{array}\right.
$$

\footnotetext{
6 Para um gás molecular ideal, $f=1$; para qualquer partícula de spin 1/2 ou partículas não massivas de $\operatorname{spin} 1$ (fóton ou o fônon), $f=2$. Esse fator indica a multiplicidade dos estados associados às partículas.
}

o número de estados acessíveis em qualquer intervalo de energia pode ser determinado integrando-se $g(\epsilon) \mathrm{em}$ relação à energia.

\section{Gases ideais não degenerados}

De acordo com os experimentos sobre o comportamento dos gases 12 15], anteriores ao século XX, a energia interna $(U)$ de um gás ideal monoatômico, em condições ambientais, à temperatura $T$ e, portanto, não degenerado, é igual a

$$
U=\frac{3}{2} N k T=\frac{3}{2} n R T .
$$

sendo $k \simeq 1,38 \times 10^{-23} \mathrm{~J} / \mathrm{K}$ a constante de Boltzmann, $R \simeq 8,3 \mathrm{~J} \cdot \mathrm{mol}^{-1} \cdot \mathrm{K}^{-1}$ a constante dos gases, $N$ o número de moléculas e $n$ o número de mols.

O calor específico molar depende dos parâmetros de um sistema como pressão $(P)$, volume $(V)$ e magnetização $(M)$, entre outros. Para um processo a volume constante é definido por 7

$$
c_{V}=\frac{1}{n}\left(\frac{\partial U}{\partial T}\right)_{V} .
$$

Assim, para um gás ideal monoatômico,

$c_{V}=\frac{3}{2} R$ (gás ideal não degenerado monoatômico).

Em baixas temperaturas, esse comportamento clássico do calor específico não é compatível com a 3 a lei da Termodinâmica [12 15], segundo a qual o calor específico a $0 \mathrm{~K}$ deve-se anular,

$$
\lim _{T \rightarrow 0} c_{V} \rightarrow 0
$$

\section{Temperaturas de degenerescência}

Uma vez que a energia média por partícula $\bar{\epsilon}=U / N$ de um gás não degenerado é da ordem de $k T$, o número de estados ocupados pelas partículas de um gás não degenerado é cerca de $G(\bar{\epsilon})$ e, portanto, o critério para a não degenerescência pode ser expresso como

$$
\bar{G} \simeq\left\{\begin{array}{l}
\left(\frac{3}{4 \pi f}\right)\left(\frac{h^{2}}{2 m k}\right)^{3 / 2}\left(\frac{N}{V}\right) \frac{1}{T^{3 / 2}} \\
\left(\frac{3}{4 \pi f}\right)\left(\frac{c h}{k}\right)^{3}\left(\frac{N}{V}\right) \frac{1}{T^{3}}
\end{array}\right.
$$

A figura 2 mostra o compromisso entre a densidade de partículas $(N / V)$ e a temperatura de um gás de partículas

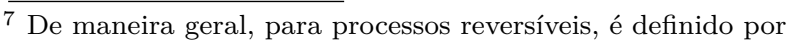

$$
c_{X}=\frac{T}{n}\left(\frac{\partial S}{\partial T}\right)_{X},
$$
}

em que $S$ é a entropia do gás, e $X$ o parâmetro mantido constante. 
massivas não relativísticas - a linha pontilhada ${ }^{8}$ define os valores que separam as regiões nas quais o gas é degenerado ou não degenerado.

Para temperaturas e densidades abaixo dessa linha, o estado do gás é degenerado e a natureza quântica de suas partículas deve ser considerada. Para temperaturas e densidades acima da linha crítica, o estado do gás é não degenerado e pode ser tratado como um sistema clássico.

A Tabela 1 mostra os valores das densidades e das temperaturas que distinguem os estados degenerados e não degenerados de alguns gases moleculares.

Em função da densidade de partículas $(N / V)$, as temperaturas de degenerescência $\left(T_{d}\right)$, definidas pela condição $N / G=1$, são dadas por

$$
T_{d}=\left\{\begin{array}{l}
T_{F}=\left(\frac{3}{4 \pi f}\right)^{2 / 3}\left(\frac{h^{2}}{2 m k}\right)\left(\frac{N}{V}\right)^{2 / 3}, \\
T_{D}=\left(\frac{3}{4 \pi f}\right)^{1 / 3}\left(\frac{c h}{k}\right)\left(\frac{N}{V}\right)^{1 / 3},
\end{array}\right.
$$

em que $T_{F}$ e $T_{D}$ são usualmente denominadas temperatura de Fermi e temperatura de Debye.

Assim, o critério para a não degenerescência de um gás é usualmente expresso como

$$
\frac{T}{T_{d}} \gg 1 \text { (critério para a não degenerescência). }
$$

Se $T \leq T_{d}$, o gás é dito degenerado.

Os gases ideais, não degenerados ou degenerados, são idealizações que não correspondem exatamente a nenhum

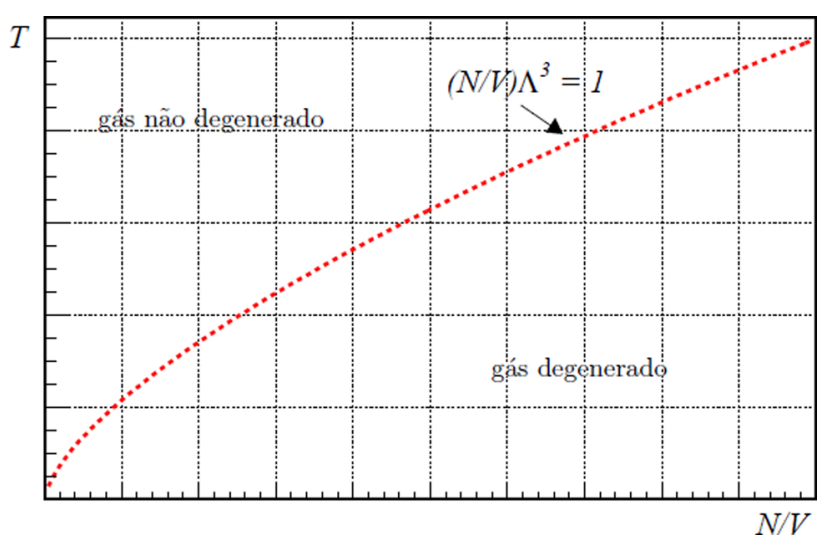

Figura 2: Limite de degenerescência dos gases.

Tabela 1: Valores da densidade e a correspondente temperatura de degenerescência de alguns gases moleculares.

\begin{tabular}{ccc}
\hline gases & $N / V\left(\mathrm{~cm}^{-3}\right)$ & $T(\mathrm{~K})$ \\
\hline $\mathrm{H}_{2}$ & $\sim 10^{19}$ & $\sim 0,1$ \\
$\mathrm{He}^{4}$ & $\sim 10^{22}$ & $\sim 3$ \\
\hline
\end{tabular}

8 Essa linha, de acordo com a primeira das eqs. 27, é definida por $(N / V) \Lambda^{3}=1$, em que $\Lambda=\left(\frac{3}{8 \pi}\right)^{1 / 3} \frac{h}{\sqrt{2 m k T}}$. sistema macroscópico. Apesar disso, a ênfase observada em seu estudo, bastante simples, decorre do fato de que em um grande número de casos alguns sistemas macroscópicos podem ser representados por conjuntos de constituintes quase independentes, ou seja, como gases ideais.

A Tabela 2 mostra, de acordo com as eqs. (8), as temperaturas de degenerescência relativas a vários sistemas que se comportam como gases ideais. Pode-se observar, assim, que, mesmo para baixas temperaturas $(T \sim 10 \mathrm{~K})$, os gases moleculares comportam-se como gases ideais não degenerados; já os elétrons de condução nos metais constituem um sistema degenerado em qualquer temperatura, uma vez que a $10^{5} \mathrm{~K}$ não existe matéria sólida. No caso dos elétrons relativísticos provenientes dos átomos de hélio ionizados de uma estrela anã branca a $10^{7} \mathrm{~K}$, a temperatura da estrela ainda é muito menor que o valor crítico e, portanto, o sistema comporta-se como um gás degenerado relativístico. Por outro lado, os elétrons em semicondutores e os osciladores atômicos nos cristais não são degenerados nas condições ambientais, sendo degenerados apenas em baixas temperaturas $(T \sim 10 \mathrm{~K})$.

Em termos das temperaturas de degenerescência, os números e as densidades de estados podem ser escritos como

$$
G(\epsilon)=\left\{\begin{array}{l}
N\left(\frac{\epsilon}{k T_{F}}\right)^{3 / 2}, \\
N\left(\frac{\epsilon}{k T_{D}}\right)^{3},
\end{array} \Longrightarrow g(\epsilon)=\left\{\begin{array}{l}
\frac{3}{2} \frac{N}{\left(k T_{F}\right)^{3 / 2}} \epsilon^{1 / 2} \\
3 \frac{N}{\left(k T_{D}\right)^{3}} \epsilon^{2}
\end{array}\right.\right.
$$

Tabela 2: Temperaturas de degenerescência de alguns sistemas típicos. Os três primeiros exemplos são de sistemas de partículas massivas não relativísticas e os dois últimos, de partículas não massivas e ultrarrelativísticas, respectivamente.

\begin{tabular}{lcc}
\hline \multicolumn{1}{c}{ sistemas } & $\begin{array}{c}\text { densidade de } \\
\text { de partículas }\left(\mathrm{cm}^{-3}\right)\end{array}$ & $\begin{array}{c}\text { temperatura } \\
T_{d}(\mathrm{~K})\end{array}$ \\
\hline $\begin{array}{l}\text { gases } \\
\text { moleculares }\end{array}$ & $10^{19}$ & $<0,1$ \\
$\begin{array}{l}\text { elétrons em } \\
\text { semicondutores }\end{array}$ & $10^{17}$ & 10 \\
$\begin{array}{l}\text { elétrons de condução } \\
\text { em metais }\end{array}$ & $10^{23}$ & $10^{5}$ \\
$\begin{array}{l}\text { osciladores atômicos } \\
\text { em cristais }\end{array}$ & $10^{23}$ & $10^{2}$ \\
$\begin{array}{l}\text { estrelas do tipo } \\
\text { anã branca }\end{array}$ & $10^{36}$ & $10^{10}$ \\
\end{tabular}




\section{Gases ideais degenerados}

O princípio de exclusão de Pauli $5,9,12$, , em sua versão original, proíbe que mais de dois elétrons atômicos ocupem o mesmo estado de energia, sendo que os dois podem ter os mesmos números quânticos exceto o de spin. A partir daí, pode-se estabelecer uma outra correlação quântica tal que partículas idênticas de spin semi-inteiro não podem compartilhar o mesmo estado. Assim, os gases ideais degenerados dividem-se em duas classes: aqueles constituídos por partículas de spin semi-inteiro, denominadas férmions, e aqueles constituídos por partículas de spin inteiro, denominadas bósons. Nos gases não degenerados, quando efetivamente não há competição pelos estados acessíveis por suas partículas constituintes, tal distinção não é necessária.

Em temperaturas próximas a $0 \mathrm{~K}$, quando o calor específico e a entropia de um sistema tendem a se anular, as partículas de um gás distribuem-se entre os estados acessíveis tal que a energia total seja mínima. Diz-se que o sistema encontra-se em seu estado fundamental.

Para temperaturas finitas, mas ainda muito menores que a temperatura crítica $\left(T \ll T_{c}\right)$, uma pequena fração das partículas do gás são excitadas além do estado fundamental. Essas partículas excitadas comportam-se, praticamente, como um subsistema não degenerado responsável pelas propriedades térmicas do gás.

\subsection{Férmions não relativísticos fortemente degenerados}

Devido ao princípio de Pauli, para um gás ideal de férmions completamente degenerado $(T=0 \mathrm{~K})$ em seu estado fundamental, as partículas se acomodam nos estados acessíveis de tal forma que cada estado seja ocupado por apenas uma partícula. Nessas condições, o número de estados ocupados é igual ao número de partículas.

Assim, para um gás de férmions não relativísticos completamente degenerado no estado fundamental,

$$
\frac{N}{G\left(\epsilon_{F}\right)}=1
$$

sendo $\epsilon_{F}$ o maior valor de energia associado aos estados ocupados pelas partículas. De acordo com as eqs. (10), esse valor, denominado energia de Fermi, é dado por

$$
\epsilon_{F}=k T_{F}
$$

Segundo a Tabela 2, para o gás de elétrons de condução de um metal à temperatura ambiente $\left(T \ll T_{F}\right)$, a energia de Fermi é da ordem de

$$
\epsilon_{F} \simeq 10^{-18} \mathrm{~J} \simeq 10 \mathrm{eV}
$$

e a energia dos elétrons excitados cerca de

$$
\epsilon \simeq k T \simeq 25 \mathrm{meV} \ll \epsilon_{F} \quad(T \sim 300 \mathrm{~K}) .
$$

Para temperaturas acima de $0 \mathrm{~K}$, mas ainda muito menores que a temperatura de Fermi $\left(T \ll T_{F}\right)$. ${ }^{9}$ algumas partículas são excitadas acima do nível de Fermi. Essas partículas excitadas comportam-se como um subsistema não degenerado com energia média por partícula da ordem de $k T \ll \epsilon_{F}$. Nessas condições, o número de partículas excitadas $\left(N_{\text {exc }}\right)$ é aproximadamente igual ao número de estados acessíveis no intervalo de energia $\left(\epsilon_{F}, \epsilon_{F}+k T\right)$, ou seja,

$$
\begin{aligned}
& N_{\mathrm{exc}} \simeq \int_{\epsilon_{F}}^{\epsilon_{F}+k T} \frac{3}{2} \frac{N}{\epsilon_{F}^{3 / 2}} \epsilon^{1 / 2} \mathrm{~d} \epsilon \\
& =\frac{N}{\epsilon_{F}^{3 / 2}}\left[\left(\epsilon_{F}+k T\right)^{3 / 2}-\epsilon_{F}^{3 / 2}\right] \\
& \simeq N\left[\left(1+k T / \epsilon_{F}\right)^{3 / 2}-1\right] \simeq \frac{3}{2} N \frac{T}{T_{F}} .
\end{aligned}
$$

Pode-se obter a energia interna $(U)$ do gás adicionandose a energia do estado fundamental $\left(U_{\circ}\right)$ para $T=0 \mathrm{~K}$ à energia das partículas excitadas $\left(N_{\text {exc }} k T\right)$,

$$
U \simeq U_{\circ}+N_{\mathrm{exc}} k T=U_{\circ}+\frac{3}{2} N k \frac{T^{2}}{T_{F}},
$$

e o calor específico molar a volume constante, por ${ }^{10}$

$$
c_{V} \simeq 3 R \frac{T}{T_{F}}\left(T \ll T_{F}\right) \text { (férmions não relativísticos). }
$$

Esse comportamento, obtido por Sommerfeld em 1928, compatível com a 3 a lei da Termodinâmica, é o esperado para a variação do calor específico dos metais em temperaturas próximas a $0 \mathrm{~K}$, quando as propriedades térmicas dos metais são atribuídas ao movimento dos elétrons de condução 13 .

\subsection{Bósons ideais degenerados}

Uma vez que os bósons de um sistema podem ser associados a estados de mesma energia, todos podem ocupar um único estado. Em geral, a energia do estado fundamental de um gás de bósons completamente degenerado $(T=0 \mathrm{~K})$ pode ser considerada nula.

De modo análogo aos férmions, para temperaturas um pouco acima de $0 \mathrm{~K}$, mas ainda muito menores que a temperatura de degenerescência $\left(T \ll T_{D}\right)$, algumas partículas são excitadas com energia da ordem de $k T$.

Dependendo da massa e do caráter relativístico, o número de partículas excitadas $\left(N_{\epsilon>0}\right)$, bem como a energia interna $(U)$ e o calor específico molar $\left(c_{V}\right)$, são calculados de modos distintos.

\footnotetext{
${ }_{9}^{9}$ Para os elétrons de condução em um metal, essa condição sempre se verifica.

10 Segundo Sommerfeld 13, $c_{V}=\frac{\pi^{2}}{2} R \frac{T}{T_{F}}$.
} 


\subsubsection{Bósons não massivos fortemente degenerados}

No caso de bósons não massivos, apesar de ser pequena a fração de partículas que abandonam o estado fundamental quando $T \ll T_{D}$, o número de partículas excitadas com energia média da ordem de $k T$ é maior do que o número de estados com energia até $k T$. Assim,

$$
N_{\epsilon>0}=\alpha \underbrace{\int_{0}^{k T} 3 \frac{N}{\left(k T_{D}\right)^{3}} \epsilon^{2} \mathrm{~d} \epsilon}_{G(\epsilon>0)}=N \alpha\left(\frac{T}{T_{D}}\right)^{3},
$$

sendo $\alpha$ um parâmetro da ordem de 10, que depende da razão $T / T_{D}[1]$

Desse modo, a energia interna corresponde a

$$
U=N_{\epsilon>0} k T=N k \alpha \frac{T^{4}}{T_{D}^{3}}
$$

e o calor específico molar a volume constante, a

$$
c_{V}=4 R \alpha\left(\frac{T}{T_{D}}\right)^{3} \quad\left(T \ll T_{D}\right) \text { ( bósons não massivos). }
$$

A dependência da temperatura $\left(\propto T^{3}\right)$, estabelecida por P. Debye 19], em 1912, é compatível com a $3^{\text {a }}$ lei da Termodinâmica, e descreve o comportamento do calor específico molar dos sólidos em baixas temperaturas $(T \sim$ 4 K) 2012

\subsubsection{Os sólidos cristalinos}

A lei empírica de Dulong e Petit, de que o valor do calor específico dos sólidos seria uma constante independente da temperatura, apesar dos muitos desvios observados, só começa a declinar, realmente, no início do século XX. A partir de misturas refrigerantes, foram alcançadas temperaturas baixas o suficiente para evidenciar a dependência do calor específico com a temperatura. Verificou-se, experimentalmente [20], que, em baixas temperaturas, o calor específico de um sólido obedecia à 3 a lei da Termodinâmica, variando com a temperatura segundo a chamada lei de Debye, eq. (15), a seguir.

A representação das vibrações atômicas nos sólidos cristalino $\sqrt{13}$ como um gás de bósons não massivos, chamados fônons, baseia-se na aproximação harmônica da energia potencial efetiva, a qual descreve as interações de cada átomo com seus vizinhos. Desse modo, cada átomo é

$11 \alpha=\pi^{4} / 5 \quad\left(T \ll T_{D}\right)$.

12 Para os sólidos, o calores específicos a volume e a pressão constantes são praticamente iguais, principalmente em baixas temperaturas.

13 Um sólido cristalino é constituído pela repetição de uma unidade básica de padrão geométrico regular, na qual os átomos executam pequenas vibrações em torno de posições relativas mais ou menos fixas. associado a um oscilador harmônico independente dos demais, cujo espectro de energia, $\epsilon_{n}(\nu)$, segundo a Mecânica Quântica, é dado por

$$
\epsilon_{n}(\nu)=(n+1 / 2) h \nu=\epsilon_{\circ}+n h \nu \quad(n=0,1, \ldots),
$$

em que $\nu$ é a frequência natural de vibração, e $\epsilon_{\circ}=h \nu / 2$ é a energia do estado fundamental de um particular oscilador.

A energia de cada átomo em um sólido cristalino em equilíbrio térmico, portanto, é composta por uma parcela constante, a energia do estado fundamental ${ }^{14}$ e uma parcela que depende do grau de excitação do átomo, ou seja, da temperatura do cristal.

Considerando que cada estado excitado de um oscilador corresponde a $n$ partículas independentes não massivas, cada qual com energia $\epsilon=h \nu$, o conjunto de osciladores que representam as vibrações atômicas do cristal pode ser associado a um sistema de partículas independentes que se comportam como um gás de bósons não massivos, pois o número $(n)$ de partículas associado a cada estado depende da temperatura e, portanto, não obedece ao princípio de exclusão de Pauli.

Em baixas temperaturas, as vibrações atômicas em um sólido cristalino são equivalentes a um gás degenerado de bósons não massivos, os fônons.

Como cada átomo corresponde a 3 osciladores independentes, o número total de osciladores no cristal é $3 N$, e o calor específico molar é dado pela lei de Debye $5,12,15,21,15$

$$
c_{V}=12 R \alpha\left(\frac{T}{T_{D}}\right)^{3} \quad\left(T \ll T_{D}\right) \quad \text { (fônons). }
$$

O resultado do experimento pioneiro de P.H. Keesom e N. Pearlman [20], de 1955, apresentado no gráfico $c_{V} / T$ versus $T^{2}$ da figura 3 mostra que o comportamento do calor específico dos sólidos não metálicos a baixas temperaturas $\left(T<T_{D} / 50 \sim 4 \mathrm{~K}\right)$ é compatível com a lei de Debye.

Em altas temperaturas $\left(T \gg T_{D}\right)$, os sólidos comportamse como um sistema não degenerado cujo calor específico molar $\left(c_{V}\right)$ obedece à lei de Dulong-Petit $2,5,12,15,21$, ,

$$
\begin{aligned}
& \mathrm{c}_{V}=3 R \simeq 6 \mathrm{cal} \cdot \mathrm{mol}^{-1} \cdot \mathrm{K}^{-1} \simeq 25 \mathrm{~J} \cdot \mathrm{mol}^{-1} . \\
& \mathrm{K}^{-1} . \\
& \left(T \gg T_{D}\right) \text { (fônons). }
\end{aligned}
$$

Tanto do ponto de vista teórico, como experimentalmente, o calor específico dos sólidos cresce suavemente com a temperatura até o valor limite dado pela lei de Dulong-Petit (figura 4 .

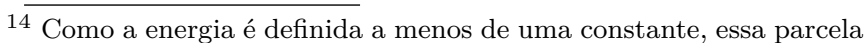
pode ser considera nula.

15 A rigor a lei de Debye é válida em temperaturas menores que $T_{D} / 50[21$.
} 


\subsubsection{O calor específico dos metais}

Os metais constituem uma classe especial de sólidos cristalinos. Além dos íons que constituem a rede cristalina, e vibram em torno de suas posições de equilíbrio, possuem também praticamente o mesmo número de elétrons, os elétrons de condução, que não estão associados a nenhum particular íon.

A hipótese de que algumas das propriedades de um metal pudessem ser obtidas a partir do modelo do gás de elétrons remontam à época de P. Drude (1900). Tal hipótese apoia-se no fato de que em um cristal os íons positivos do metal estabelecem um campo eletromagnético que tende a anular a ação dos outros elétrons sobre um determinado elétron. No entanto, ao se considerar os elétrons de condução como um gás não degenerado, os resultados obtidos não foram compatíveis com o comportamento térmico observado. Apenas quando Sommerfeld considerou-os como um gás degenerado de férmions de spin $1 / 2$, os resultados teóricos tornaram-se compatíveis com os experimentos 22 .

Assim, o calor específico de um metal possui uma componente devida às vibrações da rede, ou a um gás degenerado de fônons, e outra devida ao gás degenerado

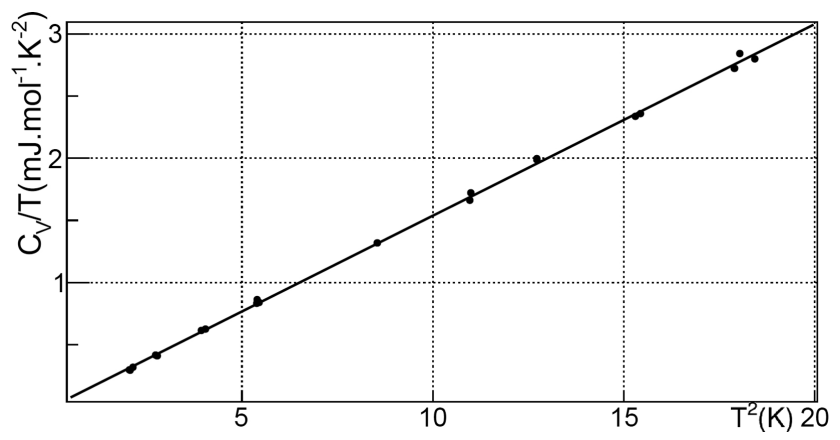

Figura 3: Calor específico molar do $\mathrm{KCl}$ em temperaturas abaixo de $4 \mathrm{~K}$. A linha contínua é a reta de ajuste aos dados (pontos) de Keesom e Pearlman, de coeficiente angular $15,4 \times 10^{-5} \mathrm{~J} \cdot \mathrm{mol}^{-1} \cdot \mathrm{K}^{-2}$. A temperatura de Debye estimada é da ordem de $(233 \pm 3) \mathrm{K}$.

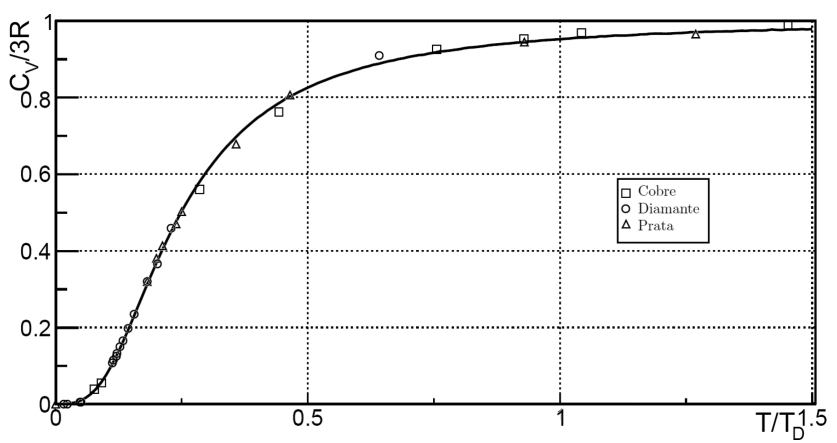

Figura 4: Comparação da curva teórica de Debye (linha contínua) com os dados experimentais (pontos) relativos ao calor específico molar de alguns sólidos [15].

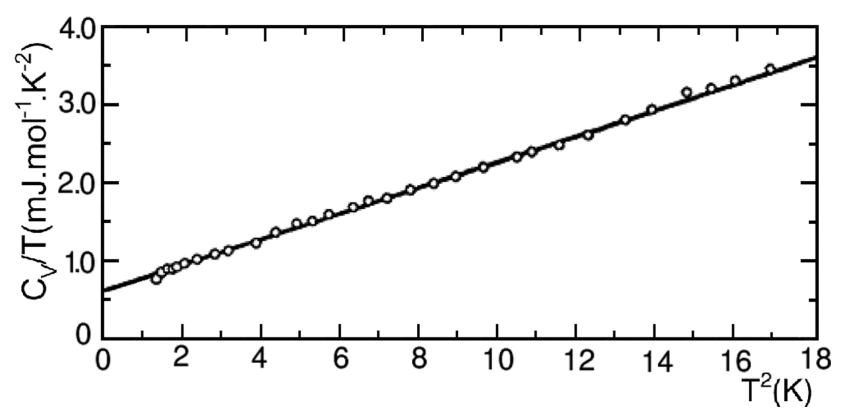

Figura 5: Calor específico de uma amostra de prata. A linha contínua é a reta de ajuste aos dados (pontos) experimentais de Corak et. al. O coeficiente linear da ordem de $0,6 \times 10^{-3} \mathrm{~J} \cdot \mathrm{mol}^{-1} \cdot \mathrm{K}^{-2}$ corresponde à temperatura de Fermi de $6,8 \times 10^{4} \mathrm{~K}$.

de elétrons de condução do metal,

$$
c_{\text {metal }}=c_{\text {fônons }}+c_{\text {elétrons }} \text {. }
$$

Como à temperatura ambiente $T \ll T_{F}$ para qualquer metal, a contribuição eletrônica só é relevante em temperaturas muito menores que a temperatura de Debye do metal, quando o calor específico pode ser expresso como

$$
c_{\text {metal }}=\alpha T^{3}+\gamma T \quad\left(T \ll T_{D}\right),
$$

em que o parâmetros $\alpha$ e $\gamma$ determinam, respectivamente, a temperatura de Debye e a temperatura de Fermi.

Esse comportamento dos metais, já observado por Keesom (1935), foi estabelecido nos experimentos realizados por W. Corak, M.P. Garfunkel, C.B. Satterthwaite e A. Wexler em 1955 22. (figura 5).

\subsubsection{A radiação de corpo negro}

De maneira análoga às vibrações, o campo eletromagnético associado à radiação de corpo negro em equilíbrio térmico pode ser representado por um gás de bósons não massivos - os fótons com energias $h \nu(\nu=0, \ldots \infty)$ [5, $12,13,15,23$.

Nas vibrações atômicas, a energia do estado fundamental a $0 \mathrm{~K}$ tem valor constante e finito determinado pelo número de osciladores do cristal; no caso da radiação de corpo negro, o número total de osciladores associados ao campo eletromagnético não é finito. Assim, para se contornar o problema de um número infinito de termos contribuir para a energia do estado fundamental do sistema a $0 \mathrm{~K}$, considera-se que a energia do sistema é a energia dos estados excitados, ou seja, a energia dos fótons.

Uma vez que não existem restrições quanto ao número de fótons, pois esse número pode ser infinito, a radiação de corpo negro sempre constitui um sistema degenerado de bósons não massivos em qualquer temperatura.

Substituindo-se a expressão para a temperatura de Debye (a segunda das eqs. (8) na eq. (13), a densidade de energia $(u=U / V)$ da radiação de corpo negro pode ser escrita na forma usual da lei de Stefan-Boltzmann 
[5, $12,13,15]$

$$
u=\frac{8 \pi \alpha}{3} \frac{k^{4}}{(c h)^{3}} T^{4}=a T^{4}
$$

sendo $a \simeq 7,6 \times 10^{-16} \mathrm{~J} \cdot \mathrm{m}^{-3} \cdot \mathrm{K}^{-4}$, para $\alpha=\pi^{4} / 5$.

\subsubsection{Bósons massivos não relativísticos fortemente degenerados e condensação de Bose-Einstein}

O número de partículas excitadas em um gás de bósons é diferente quando eles são massivos ou não, devido, principalmente, ao fenômeno de criação e aniquilação de partículas. Tanto a energia da radiação eletromagnética de corpo negro, quanto das oscilações atômicas nos cristais, podem ser descritas pela soma das energias de bósons não massivos, respectivamente denominados fótons e fônons. Uma vez que a quantidade desses bósons não massivos não é fixa, dependendo fortemente da temperatura $\left(\propto T^{3}\right)$, diz-se que fótons e fônons podem ser criados ou aniquilados.

Diferentemente dos bósons não massivos, os bósons massivos não relativísticos não podem ser criados ou aniquilados, o que implica conservação do número de partículas ${ }^{16}$ Desse modo, a energia do estado fundamental é nula e pode-se admitir que em baixas temperaturas o número de partículas excitadas com energia média da ordem de $k T$ seja dado pelo número de estados com energia até $k T{ }^{17}$

$$
N_{\epsilon>0}=\int_{0}^{k T} \frac{3}{2} \frac{N}{\left(k T_{d}\right)^{3 / 2}} \epsilon^{1 / 2} \mathrm{~d} \epsilon=N\left(\frac{T}{T_{d}}\right)^{3 / 2},
$$

e a energia interna e o calor específico molar a volume constante, compatível com a 3 a lei da Termodinâmica, sejam, aproximadamente,

$$
U \simeq N_{\epsilon>0} k T=N k \frac{T^{5 / 2}}{T_{d}^{3 / 2}}, \quad \text { e } \quad c_{V} \simeq R\left(\frac{T}{T_{d}}\right)^{3 / 2} .
$$

O número de bósons no estado fundamental $\left(N_{\circ}=\right.$ $N-N_{\epsilon>0}$ ), por sua vez, é dado por

$$
N_{\circ}=N\left[1-\left(\frac{T}{T_{d}}\right)^{3 / 2}\right]
$$

De acordo com a eq. (19), abaixo da temperatura de degenerescência o gás se aproxima de um estado em que o número de bósons massivos agrupados nas vizinhanças do estado fundamental cresce rapidamente (figura 6). Com base nesse comportamento, Einstein supõe que nessa

\footnotetext{
16 No caso de bósons massivos relativísticos, deve-se considerar também os processos de criação e aniquilação.

17 A temperatura de degenerescência e a densidade de estados para bósons massivos não relativísticos são calculadas do mesmo modo que para os férmions não relativísticos, pois a relação entre a energia $(\epsilon)$ e o momentum $(p)$ de partículas não relativísticas de massa $m$ é dada por $p=\sqrt{2 m \epsilon}$.
}

temperatura ocorra um novo fenômeno, no qual o sistema de bósons massivos atinja um estado mais organizado da matéria, em uma transição do tipo desordem-ordem. Em analogia com a condensação de um gás ordinário, o fenômeno é conhecido, desde então, como condensação de Bose-Einstein 24 26 e, nesse caso, a temperatura de degenerescência é uma temperatura crítica $T_{c}$ que caracteriza uma transição de fase.

Sendo nula a energia do estado fundamental, a grande maioria das partículas do chamado condensado de BoseEinstein possui momentum nulo em baixas temperatu$\operatorname{ras}\left(T / T_{c}<0,4\right)$. Em maior número que as demais, tais partículas contribuiriam com maior peso para as propriedades macroscópicas do sistema, como pressão e viscosidade.

Essas conclusões foram estabelecidas por Einstein em 1924 e 1925 27, 28, a partir do método de contagem de fótons da radiação de corpo negro utilizado por Bose. Bose considerou que os fótons não obedeciam ao princípio de Pauli, à época desconhecido, e eram indistinguiveis 18 Ao estender o procedimento de Bose aos gases moleculares, Einstein obtém uma teoria quântica dos gases ideais degenerados de bósons massivos não relativísticos.

A descoberta da existência de um novo estado condensado da matéria foi considerada, inicialmente, apenas um resultado matemático, sem possibilidade de verificação. Devido ao baixíssimo valor da temperatura crítica de um gás molecular $\left(T_{c}<0,1 \mathrm{~K}\right)$, qualquer gás real a tão baixa temperatura estaria no estado líquido.

Apesar dos argumentos contrários à condensação de Bose-Einstein, F. London, em 1938, estabelece que o calor específico molar a volume constante de um gás de bósons massivos não relativísticos para temperaturas até o valor crítico é dado por 24

$$
c_{V}=1,93 R\left(\frac{T}{T_{c}}\right)^{3 / 2} \quad\left(T \leq T_{c}\right)
$$

(bósons massivos não relativísticos).

Como o valor do calor específico molar à temperatura crítica $(1,93 R)$ excede o valor clássico $(1,5 R)$ para o qual deve se aproximar assintoticamente para $T>T_{c}$, nas vizinhanças da temperatura crítica o calor específico do

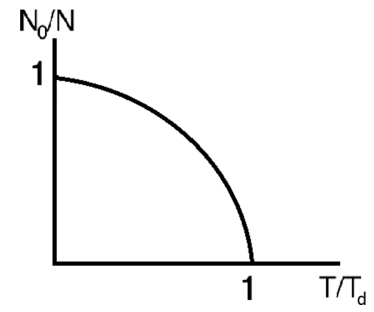

Figura 6: Número de bósons não massivos no condensado de Bose-Einstein.

18 Bose também admitiu corretamente que, devido a polarização da luz, o fator de multiplicação de estados era igual a $2(f=2)$. 
gás apresenta um comportamento não suave (figura 7), o que implica descontinuidade em sua derivada.

Foi exatamente a partir desse comportamento que London defendeu a hipótese de Einstein sobre a temperatura crítica de um gás de bósons massivos não relativísticos estar associada a uma transição de fase. Rebatendo as críticas de que o fenômeno da condensação de BoseEinstein resultaria de um artifício matemático, London sugere a transição líquido-superfluido do hélio $\left(\mathrm{He}^{4}\right)$ como um exemplo de condensação de Bose-Einstein que ocorreria na natureza 19

\subsection{6. $\mathrm{O} \mathrm{He}^{4}$ líquido}

A particularidade mais marcante do $\mathrm{He}^{4}$ líquido $(T \sim 4 \mathrm{~K})$ quando é resfriado em equilíbrio com sua pressão de vapor, após alcançar a temperatura crítica da ordem de $2 \mathrm{~K}$, é a súbita capacidade de fluir em tubos capilares sem exercer pressão, como se deixasse de ter viscosidade. Essa mudança brusca na viscosidade, acompanhada também de uma variação brusca no calor específico, é a propriedade que caracteriza o fenômeno como uma transição de fase líquido-superfluido.

Uma vez que os átomos de $\mathrm{He}^{4}$ possuem seis partículas (2 prótons, 2 nêutrons e 2 elétrons) de spin semi-inteiros, F. London supõe, em primeira aproximação, que o hélio líquido constituído dos isótopos $\mathrm{He}^{4}$ pode ser considerado um sistema de partículas independentes de spin inteiros, ou seja, como um gás de bósons massivos não relativísticos, e que a transição líquido-superfluido tivesse relação com o fenômeno de condensação de Bose-Einstein.

Essa hipótese é reforçada quando, ao considerar-se $\mathrm{He}^{4}$ líquido como um gás de bósons, o valor da temperatura crítica é cerca de $3 \mathrm{~K}$, conforme Tabela 1. Apesar da

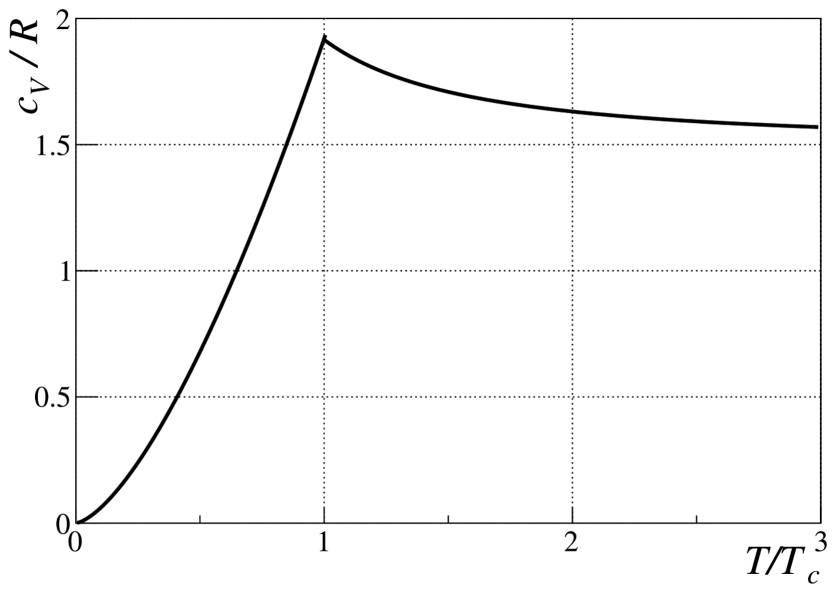

Figura 7: Calor específico de um gás de bósons massivos não relativísticos.

\footnotetext{
19 Apesar de ocorrer também a concentração de partículas no estado fundamental de um gás de bósons não massivos em baixas temperaturas, a energia do estado fundamental não é nula e o calor específico varia suavemente (figura 4, não havendo descontinuidade em sua derivada e, portanto, não caracterizando uma transição de fase.
}

similaridade e do estabelecimento posterior do fenômeno como um exemplo de condensação de Bose-Einstein 20 por não ser exatamente um sistema de partículas que não interagem, o comportamento do calor específico do $\mathrm{He}^{4}$ líquido é muito diferente do gás ideal degenerado de bósons massivos.

A procura de um sistema gasoso que exibisse a condensação de Bose-Einstein só teve êxito em 1995, quando vapores de átomos de $\mathrm{Rb}$, a uma densidade de $10^{12} \mathrm{~cm}^{-3}$, foram resfriados a baixíssima temperatura, cerca de 100 nK, por um grupo do JILA ${ }^{21}$ Desde então, vários experimentos sobre esse estranho comportamento da matéria foram realizados, constituindo-se em uma ativa área de pesquisa da Física de ultra baixas temperaturas 26, 29.

\section{Considerações finais}

Do ponto de vista dinâmico, ainda que em equilíbrio térmico, o número de partículas associado a cada nível de energia $(\epsilon)$ de um gás varia incessantemente. Devido a essas flutuações e ao grande número de partículas, a distribuição das partículas pelos estados associados aos níveis de energia do gás são caracterizadas pelos números médios de ocupação, $\left\langle n_{\epsilon}\right\rangle$, ou população média dos estados.

A distribuição das partículas depende do estado de degenerescência do gás e, portanto, da natureza de suas partículas constituintes. Além das correlações decorrentes do princípio de exclusão de Pauli, deve-se considerar que as partículas, bósons e férmions, de um gás ideal são, entre si, indistinguíveis.

As três distribuições usuais para os gases ideais podem ser sintetizadas como 12,13

$$
\left\langle n_{\epsilon}\right\rangle=\frac{1}{\lambda^{-1} e^{\epsilon / k T}+a}
$$

em que $\lambda$ é a fugacidade do gás 22

A distribuição de Maxwell-Boltzmann $(a=0)$ descreve o comportamento da população média em um gás ideal não degenerado, a distribuição de Fermi-Dirac $(a=1)$, a população média em um gás ideal de férmions, e a distribuição de Bose-Einstein $(a=-1)$, a população média em gases ideais de bósons não massivos $(\lambda=1)$ e massivos 23

20 A observação da população de átomos de $\mathrm{He}^{4}$ com momentum nulo foi realizada em 1982 26].

21 Joint Institute for Laboratoty Astrophysics, um instituto conjunto da University of Colorado e do NIST (National Institute of Standards and Technology).

22 Para partículas massivas a fugacidade $(\lambda)$ é determinada pela restrição ao número de partículas; para bósons não massivos não há restrição associada ao número de partículas, e no caso dos fônons, o espectro de energia tem um limite determinado pela temperatura de Debye $\left(\epsilon_{\max }=k T_{D}\right)$.

23 Para bósons não massivos, principalmente, para fótons da radiação de corpo negro, a distribuição é chamada também distribuição de Planck. 
Assim, as propriedades macroscópicas de um gás em equilíbrio térmico, como a energia média $U$, ou energia interna, e o número total de partículas $N$, satisfazem as seguintes relações

$$
\left\{\begin{array}{l}
U=\int_{0}^{\infty} \epsilon g(\epsilon)\left\langle n_{\epsilon}\right\rangle \mathrm{d} \epsilon \\
N=\int_{0}^{\infty} g(\epsilon)\left\langle n_{\epsilon}\right\rangle \mathrm{d} \epsilon
\end{array}\right.
$$

sendo $g(\epsilon)$ a densidade de estados.

A partir dessas expressões, eqs. 22, são obtidas estimativas mais acuradas sobre o comportamento dos gases, correspondentes a um amplo domínio de temperaturas, desde as mais baixas $\left(T \ll T_{d}\right)$ às mais altas $\left(T \gg T_{d}\right)$.

\section{Referências}

[1] D. McKie and N.H. de V. Heathcote, The Discovery of Specific and Latent Heats (Edward Arnols, London, 1935).

[2] A.T. Petit and P.L. Dulong, Annales de Chimie et de Physique 10, 395 (1819).

[3] J.J. Berzelius, Annals of Philosophy 2, 443 (1813).

[4] D. Mendeleiev, Journal of the Russian Physical Chemical Society 1, 60 (1869).

[5] F. Caruso e V. Oguri, Física Moderna: Origens Clássicas e Fundamentos Quânticos (LTC, Rio de Janeiro, 2016), $2^{\mathrm{a}}$ ed.

[6] L. Boltzmann, Lectures on Gas Theory: 1896-1898 (Dover Pub. Inc., New York, 1995).

[7] J.W. Gibbs, Elementary Principles in Statistical Mechanics: 1902 (Dover Pub. Inc., New York, 2014).

[8] M. Planck and H. Kangro, Planck's Original Papers in Quantum Physics: German and English Edition (Taylor \& Francis Ltd., London, 1972).

[9] P.A.M. Dirac, The Principles of Quantum Mechanics: 1930 (Oxford University Press, Oxford, 1958), 4th ed.

[10] J. Wilks, The Third Law of Thermodynamics (Oxford University Press, Oxford, 1961).

[11] L. Masanes and J. Oppenheim, Nature Communications 8, 14538 (2017).

[12] E. Fermi, Molecules, Crystals, and Quantum Statistics (W.A. Benjamin Inc., New York, 1966).

[13] A. Sommerfeld, Thermodynamics and Statistical Mechanics, Lectures on Theoretical Physics, vol. V, (Academic Press, New York, 1955).

[14] M.W. Zemansky and R.H. Diettman, Heat and Thermodynamics (McGraw-Hill, New York, 1997), 7th ed.

[15] M.J. de Oliveira, Termodinâmica (Livraria da Física, São Paulo, 2012), $2^{\mathrm{a}}$ ed.

[16] G.I. Epifanov, Solid State Physics (Mir Publisher, Moscow, 1979).

[17] W. Grimus, arXiv:1112.3748v2.

[18] S. Bose, Revista Brasileira de Ensino de Física 27, 463 (2005), traduzido por F. Caruso e V. Oguri.

[19] P. Debye, Annalen der Physik 39, 789 (1912).

[20] P.H. Keesom and N. Pearlman, Physical Review 98, 1699 (1955).

[21] M. Blackman, Reports Progress in Phys. 8, 11 (1941).
[22] W. Corak, M.P. Garfunkel, C.B. Satterthwaite and A. Wexler, Physical Review 98, 1699 (1955).

[23] N. Studart, Revista Brasileira de Ensino de Física 22, $523(2000)$.

[24] F. London, Physical Review 54, 947 (1938).

[25] S.R. Dahmen, Revista Brasileira de Ensino de Física 27, 283 (2005).

[26] V.S. Bagnato, Revista Brasileira de Ensino de Física 19, 11 (1997).

[27] A. Einstein, http://www.thphys.uni-heidelberg de/ amendola/otherstuff/einstein-paper-v2. pdf acessado em 26/04/2017, traduzido por L. Amendola.

[28] A. Einstein, Revista Brasileira de Ensino de Física 27, 113 (2005), traduzido por S.R. Dahmen.

[29] E.A.L. Henn, J.A. Seman, G.B. Seco, E.P. Olimpio, P. Castilho, G. Roati, D.V. Magalhães, K.M.F. Magalhães and V.S. Bagnato, Brazilian Journal of Physics 38, 279 (2008). 